\title{
Increased matriptase zymogen activation by UV irradiation protects keratinocyte from cell
} death

Chi-Yung Chen ${ }^{\mathrm{a}}$, Cheng-Jueng Chen ${ }^{\mathrm{b}}$, Chih-Hsin Lai ${ }^{\mathrm{c}, \mathrm{d}}$, Bai-Yao Wu ${ }^{\mathrm{e}}$, Shiao-Pieng Lee ${ }^{\mathrm{f}}$, Michael D. Johnson ${ }^{\mathrm{g}}$, Chen-Yong Lin ${ }^{\mathrm{g}}$, and Jehng-Kang Wang ${ }^{\mathrm{a}, \mathrm{h}, *}$

${ }^{a}$ Graduate Institute of Life Sciences, National Defense Medical Center, Taipei, Taiwan, ROC

${ }^{\mathrm{b}}$ Division of General Surgery, Tri-Service General Hospital, National Defense Medical Center, Taipei, Taiwan, ROC

${ }^{c}$ Graduate Institute of Medical Sciences, National Defense Medical Center, Taipei, Taiwan, ROC

${ }^{\mathrm{d}}$ Department of Dentistry Heping Fuyou Branch, Taipei City Hospital, Taipei, Taiwan, ROC

${ }^{\mathrm{e}}$ Department of Dermatology, Tri-Service General Hospital, National Defense Medical Center, Taipei, Taiwan, ROC

${ }^{\mathrm{f}}$ School of Dentistry, National Defense Medical Center, Taipei, Taiwan, ROC

${ }^{g}$ Lombardi Comprehensive Cancer Center, Department of Oncology, Georgetown University

Washington DC 20057

${ }^{\mathrm{h}}$ Department of Biochemistry, National Defense Medical Center, Taipei, Taiwan, ROC

*Corresponding author:

Jehng-Kang Wang, Ph.D. Department of Biochemistry, National Defense Medical Center, No. 161, Sec. 6, Ming-Chung E. Rd., Taipei, 114, Taiwan, R.O.C. Email address:

jkwang@ndmctsgh.edu.tw. Tel: +886-2-87870891

This study was supported by National Cancer Institute (NCI) Grants RO1 CA 123223 (to M. Johnson and C.-Y. Lin), Taiwan Department of Defense Grant 103-M017, MAB-104-28 (to C.-J. Chen), and 103-M018, MAB-104-26, MOST 103-2320-B-016-022 (to J.-K. Wang), and Taipei Institute of Pathology Grant TIP10205 (to C.-H. Lai).

The authors have no conflict of interest to declare.

Text word: 7679; number of references: 53; tables: 1; figures: 7

\section{Keywords:}

apoptosis, epidermal regeneration, matriptase, ROS, UV irradiation.

\begin{abstract}
Abbreviation:
DTT, DL-Dithiothreitol; HAI-1, hepatocyte growth factor activator inhibitor-1; H\&E, hematoxylin and eosin; mAb, monoclonal antibody; MTP KD, matriptase knockdown; NT, nontarget; PARP, poly ADP ribose polymerase; PBN, N-tert-Butyl- $\alpha$-phenylnitrone; ROS, reactive oxygen species; UV, ultraviolet; UVA/UVB, ultraviolet light A/ ultraviolet light B; UVR, ultraviolet radiation
\end{abstract}




\section{Abstract}

Background: Overexposure to ultraviolet (UV) derived from solar light causes skin damage by causing DNA lesions and the generation of reactive oxygen species (ROS) in keratinocytes and other epidermal cells. The type 2 transmembrane serine protease matriptase has characteristics that allow keratinocytes to respond to/recover from, environmental insults to the skin. This response may depend on its roles in epidermal proliferation and early differentiation, and its rapid activation in response to changes in the cellular chemical milieu, including increased oxidative stress.

Objective: We investigate the regulation of matriptase activation and its role in the response of the skin to exposure to different parts of the UV spectrum including UVA UVB, and UVR. Methods: The activation state and distribution of matriptase in ex vivo UV exposed human skin specimens and sun damaged skin samples were analyzed by immunohistochemistry. HaCaT immortalized human keratinocytes were also used to investigate the mechanism of matriptase zymogen activation induced by UV irradiation. Levels of cytosolic ROS were determined by $\mathrm{H}_{2} \mathrm{DCF}$ assay. Activated matriptase, PARP and caspase 3 cleavage was analyzed by Western blotting, and the apoptotic ratio was measured by Hoechst 33258 staining.

Results: UVB exposure rapidly increased matriptase zymogen activation in the basal keratinocytes of skin samples. Activated matriptase was also detected at much higher levels in both the basal and spinous layer keratinocytes in sun damaged skin with actinic elastosis. UVB and solar light-induced matriptase zymogen activation likely results from UV-induced ROS generation, given that UVR, UVA, and UVB irradiation induced HaCaT human keratinocytes to activate matriptase in a dose- and time-dependent manner, and that this was suppressed by the ROS scavenger N-tert-Butyl- $\alpha$-phenylnitrone and reducing agent dithiothreitol. Matriptase deficient HaCaT keratinocytes were more susceptible to UV-induced apoptosis than control cells, suggesting a protective role for matriptase in UV exposed keratinocytes.

Conclusion: UV irradiation/ROS induced matriptase proteolysis may have short term protective effects and contribute to the recovery from acute epidermal damage and/or pathology of skin with chronic sun damage. 


\section{Introduction}

The skin acts as the first defensive barrier protecting the body against the harmful effects of environmental factors such as pathogens, toxic chemicals, physical damage, and electromagnetic radiation. Skin integrity is often damaged by these environmental insults and requires timely repair and regeneration in order to continue to perform its protective function. Solar ultraviolet (UV) light is one of the most common hazards causing skin impairment. DNA damage and the generation of reactive oxygen species (ROS) are the two major molecular events associated with exposure to UV irradiation [1]. Around one day after UV irradiation, the keratinocytes that have acquired significant DNA lesions will undergo apoptotic cell death to form the characteristic sunburn cells [2,3]. Keratinocyte differentiation is temporarily altered with the progressive disappearance and then reappearance of the differentiation marker keratin 10. The expression of some terminal differentiation markers, such as loricrin, filaggrin, transgultaminase I is also temporarily lost though others, such as involucrin and spr1, are not affected by UV irradiation [3]. Keratinocyte proliferation rate also increases transiently prior to the return of normal epidermal differentiation. Thus, UV irradiation affects epidermal morphogenesis and regeneration by selectivity altering some programs of keratinocyte differentiation.

Epidermal regeneration and morphogenesis requires keratinocyte differentiation to proceed in a precisely controlled manner for the formation of a structured and organized epithelium that provides efficient barrier function [4,5]. Tightly regulated proteolysis has been shown to play an important role in the maintenance of normal skin differentiation by virtue of the many skin disorders that are known to be caused by defects in proteases and protease inhibitors 
[6-18]. Among the proteases and protease inhibitors shown to be involved in normal epidermal differentiation, are the type 2 transmembrane serine protease matriptase and its cognate inhibitor hepatocyte growth factor activator inhibitor (HAI)-1. Several pieces of evidence suggest that matriptase may have an important role in the skin response to, and recovery from, UV irradiation. Matriptase is expressed at high levels, primarily as an inactive zymogen, on the cell-cell junctions of the keratinocytes in the basal and spinous layers of human skin, where matriptase has been shown to regulate keratinocyte proliferation and early differentiation [19]. The zymogen form of matriptase can be rapidly converted to its active form in response to changes in the cellular chemical environment, including $\mathrm{pH}$ and oxidative stress [20]. The production of reactive oxygen species is one of the features of UV toxicity and so matriptase activity is, therefore, likely to be enhanced by UV irradiation and so may contribute to the epidermal recovery from UV irradiation. Conversely, matriptase overexpression, and by extension enhanced matriptase activity, has been shown to act as a potent oncogene in the skin of an animal model system [21], and so inappropriate and/or prolonged matriptase activity might contribute to the development of squamous cell carcinoma. Chronic UV exposure to the skin causes epidermal hyperplasia and paves the way for the development of skin tumors [22]. A detailed understanding of how UV irradiation can impact matriptase function is, therefore, important to enable evaluation of the potential pathological role of matriptase in UV-induced skin cancer.

Matriptase is synthesized as an inactive protease zymogen and in human skin matriptase zymogen activation is tightly regulated and occurs at a low level, primarily in the basal keratinocytes. Increased matriptase zymogen activation in the basal layer has been observed near to areas of inflammation in several distinct human skin diseases, and increased concentrations of reactive oxygen species (ROS) from inflammatory processes has been 
suggested to be one of the mechanisms for the increased matriptase activation [23].

Furthermore, matriptase has been shown to enhance the ability of cells to survive the toxicity associated with exposure to cadmium chloride, much of the toxicity of which is attributed to oxidative stress [20]. Given these data and the fact that ROS accumulation is commonly associated with solar damage to the skin [24], in the current study we set out to investigate how UV irradiation affects matriptase zymogen activation and the role of matriptase in the fate of keratinocyte exposed to UV irradiation.

\section{Materials and Methods}

\section{Chemicals and reagents}

2'-(4-hydroxyphenyl)-5-(4-methyl-1-piperazinyl)-2,5'-bi-1H-benzimidazole

trihydrochloride hydrate, bisBenzimide (Hoechst 33258), and 5,5'-Dithio-bis-(2-Nitrobenzoic Acid) (DTNB) and 2',7'-Dichlorofluorescin diacetate ( $\left.\mathrm{H}_{2} \mathrm{DCF}-\mathrm{DA}\right)$ were purchased from SigmaAldrich (St. Louis, MO); N-tert-Butyl- $\alpha$-phenylnitrone (PBN) was obtained from Merck Millipore (Darmstadt, Germany); DL-dithiothreitol (DTT) was obtained from Amresco Inc. (Solon, $\mathrm{OH})$.

\section{Cell culture}

HaCaT immortal human keratinocytes from CLS Cell Lines Service GmbH (Eppelheim, Germany) were maintained in Dulbecco's Modified Eagle Medium (Invitrogen, Grand Island, NY) supplemented with 10\% fetal bovine serum (Invitrogen, Grand Island, NY) and incubated at $37^{\circ} \mathrm{C}$ in a humidified incubator with $5 \% \mathrm{CO}_{2}$. 


\section{Western blotting}

The cells were lysed in $50 \mathrm{mM}$ Tris $\mathrm{pH} 7.4$ buffer containing $1 \%$ Triton X-100, $1 \mathrm{mM}$ EDTA, $150 \mathrm{mM} \mathrm{NaCl}$, aprotinin $(1 \mu \mathrm{g} / \mathrm{ml})$, leupeptin $(1 \mu \mathrm{g} / \mathrm{ml}), 1 \mathrm{mMNa}_{3} \mathrm{VO}_{4}$, and $10 \mathrm{mM}$ $\mathrm{Na}_{4} \mathrm{P}_{2} \mathrm{O}_{7}$. DTNB at $1 \mathrm{mM}$ was also added to the lysis buffer used for matriptase analysis. Equal amounts of proteins were resolved by SDS-PAGE with DTT in the sample buffer and the samples being heated (reduced/heat-treated conditions) or with no DTT or sample heating (nonreducing and non-heat-treated conditions) for matriptase and HAI-1analysis. The proteins were transferred from the gels to nitrocellulose membranes which were probed with primary antibodies as described in each experiment, followed by horseradish peroxidase (HRP)conjugated secondary antibodies, and visualized using Immobilon Western Chemiluminescent HRP Substrate (Millipore, Billerica, MA) and x-ray file.

\section{UV irradiation}

Exposure of the cells to UVA (330-370 $\mathrm{nm}$ with a peak emission at $365 \mathrm{~nm}$ ) and UVB (290-320 nm with a peak emission at $312 \mathrm{~nm}$ ) was achieved using T-8L and T-8M UV-emitting fluorescent lamps (Vilber Lourmat, Torcy, France), respectively. These lamps were installed in the UV CROSS-LINKER CL-508 (UVItec Limited, Cambridge, UK) which can measure the intensity of the UV energy delivered. The power of the illumination was $2.5 \mathrm{~mW} / \mathrm{cm}^{2}$. Besides, as a similar published paper [25], UVR exposure was achieved using a GVM 20 transilluminator (Syngene, Cambridge, UK) and a PVC film to screen out wavelengths $<290 \mathrm{~nm}$. The intensity was measured using a UVX-radiometer equipped with a UVX-31 sensor (UVP Inc., Cambridge, UK) and the output power was about $8 \mathrm{~mW} / \mathrm{cm}^{2}$. Confluent monolayers of keratinocytes were covered with a thin layer of phosphate-buffered saline (PBS) for UV exposure. Following UV 
irradiation, the cells were maintained in serum-free DMEM for the indicated times at $37^{\circ} \mathrm{C}$ and lysed for western blotting analysis or ROS detection. The dose of UV irradiation was a function of exposure time and the power of the UV sources and is expressed as $\mathrm{mJ}$ delivered per square centimeter. The skin specimens were washed with PBS and cut into two parts which were either exposed to $300 \mathrm{~mJ} / \mathrm{cm}^{2}$ UVB irradiation (exposure time: 120 seconds) or given a sham exposure (negative control). After irradiation the skin specimen were maintained at $37^{\circ} \mathrm{C}$ for 10 minutes. The skin samples were then frozen in Tissue-Tek O.C.T. Compound (Sakura Finetek, Torrance, CA) for the preparation of frozen sections and immunohistochemistry. The skin specimen exposure experiments were conducted a total of three times.

\section{Immunohistochemistry}

Frozen sections of human sun-damaged skin and sham or UVB exposed normal human skin were stained using the total matriptase mAbs M24 or M32, the activated matriptase mAb M69, the HAI-1 mAb M19, or normal mouse IgG as a negative control, as described previously $[19,23]$.

\section{ROS detection}

HaCaT cells were exposed to UV irradiation as indicated, after which the medium changed to DMEM containing $15 \mu \mathrm{M}$ of $\mathrm{H}_{2}$ DCF-DA and the cells were incubated at $37{ }^{\circ} \mathrm{C}$ for 15 minutes. The cells were then washed and lysed with PBS containing 1\% Triton X-100, and fluorescence intensity (Ex: $485 \mathrm{~nm}$; Em: $538 \mathrm{~nm}$ ) per unit protein was measured by a Fluoroskan Ascent FL Luminescence Scanner (Thermo scientific, Waltham, MA). Intracellular ROS levels 
are presented as fold change in fluorescence intensity (normalized to protein concentration) relative to the level in sham exposed cells.

\section{Apoptosis detection}

HaCaT cells were harvested (including any floating cells) and fixed with $4 \%$ paraformaldehyde for 10 minutes at room temperature. The cells were then mounted on SUPERFROST PLUS slides (Thermo scientific, Waltham, MA), and stained with $24 \mu \mathrm{g} / \mathrm{ml}$ Hoechst 33258 dye. The stained cells were assessed for apoptotic changes using a Leica DM2500 fluorescence microscope (Leica, Wetzlar, Germany) at $350 \mathrm{~nm}$ excitation and $461 \mathrm{~nm}$ emission. The apoptosis ratio was calculated based on the evaluation of a total of 300 cells for each observation.

\section{Antibodies}

Rabbit polyclonal antibodies, anti-caspase 3 (\#9662), anti-cleaved-caspase 3 (\#9664), and anti-PARP (\#9542), were purchased from Cell Signaling Technology, Inc. (Danvers, MA) and used for immunoblotting by dilution at ratio of 1:500, 1:500, and 1:2000, respectively. Mouse monoclonal anti-actin antibody (MAB 1501) was obtained from Millipore (Billerica, MA) and diluted 1:8000 for immunoblotting. In addition, human matriptase monoclonal antibodies (M24 and M69) and human HAI-1 monoclonal antibody (M19) were used to detect total matriptase, activated matriptase, and HAI-1, respectively as previously described [19,23].

\section{Ethics statement}


Fresh normal human skin specimens and human sun-damaged skin samples were obtained from the Department of Dermatology, Tri-Service General Hospital, National Defense Medical Center under IRB 099-05-019 with written informed consent, approved by the TriService General Hospital Institutional Review Board.

\section{Results}

\section{Acute UVB irradiation increases matriptase zymogen activation in}

\section{normal human skin.}

Matriptase is synthesized as a zymogen and matriptase activity is regulated posttranslationally by zymogen activation, a process that converts the inactive matriptase zymogen into an active enzyme. This process can be evaluated by immunohistochemistry using a monoclonal antibody (mAb) that is specific for the activated form of matriptase (M69). We began our study of the role of matriptase in the response of the skin to UV irradiation by examining the effect of UVB on matriptase zymogen activation in samples of normal human skin in an ex vivo exposure model (Figure 1). We chose to start with UVB since it is considered to be the most important part of the UV spectrum in sunburn cell formation in the sun-damaged skin. Two Freshly prepared samples of the same human skin specimen were either exposed to UVB $\left(300 \mathrm{~mJ} / \mathrm{cm}^{2}\right.$ with total exposure time of 120 seconds), or sham exposed, after which both samples were incubated at $37^{\circ} \mathrm{C}$ for ten minutes. The samples were then frozen in OTC and frozen sections were prepared and stained with a mAb that recognizes total matriptase, or a mAb specific for the activated form of the enzyme. As previously reported [19,23], total matriptase 
was detected at high levels at cell-cell junctions in both the basal and spinous layers of the skin (Figure 1, C and G). In sham exposed skin, activated matriptase was not detected in any layer of the skin (Figure 1, D and D'), whereas in contrast, activated matriptase was detected, primarily in the basal layer sink cells in the samples exposed to UVB (Figure 1, $\mathrm{H}$ and $\mathrm{H}^{\prime}$ ). High power images, revealed that the activated matriptase was primarily located on the cell membrane (Figure 1, H'). These data suggest that the activation of human skin matriptase s rapidly induced in respond to acute UVB irradiation.

\section{UV exposure induces ROS-mediated matriptase zymogen activation}

\section{in HaCaT human keratinocytes.}

We next used the immortalized human keratinocyte cell line HaCaT as a model system to investigate the mechanism by which UV irradiation induces matriptase zymogen activation and the role of matriptase in the impact that UV irradiation has on keratinocytes. Exposing these cells to increasing doses of UVB, we found that matriptase zymogen activation was first observed after a dose of $40 \mathrm{~mJ} / \mathrm{cm}^{2}$ (delivered over 16 seconds) and that the induction of activation was maximally stimulated by $80 \mathrm{~mJ} / \mathrm{cm}^{2}$ (delivered over 32 seconds). As we have described previously, once activated, matriptase is almost immediately inactivated by the formation of a high affinity complex of the active matriptase with the inhibitor HAI-1 [26]. As a result, matriptase zymogen activation can be assessed by the appearance of the $120-\mathrm{kDa}$ matriptase-HAI-1 complex which can be detected both by the total matriptase mAb (Figure 2 A, lower panel) and by the activated form-specific matriptase mAb (Figure 2A, upper panel). We also measured the levels of cellular ROS after UVB exposure and found that ROS levels 
increased with the increasing dose of UVB (Figure 2B), with a very similar dose response profile to that observed for the induction of matriptase zymogen activation.

We next studied the kinetics of the induction of matriptase zymogen activation and ROS generation caused by exposure to $100 \mathrm{~mJ} / \mathrm{cm}^{2}$ UVB irradiation (delivered over 40 seconds). It took approximately $3 \mathrm{hr}$ post UV exposure for the onset of matriptase activation to be detected, and about $6 \mathrm{hr}$ for the increase in matriptase activation to plateau (Figure 2C). ROS levels were significantly increased $6 \mathrm{hr}$ after UVB treatment (Figure 2D), though ROS levels appeared to be increasing at the earlier time points. ROS levels were still increasing $12 \mathrm{hr}$ post UVB exposure (Figure 2D). The kinetics of matriptase zymogen activation and ROS generation appear to mirror each other, although the increase in ROS levels were detectable before matriptase activation and continued after matriptase activation had plateaued. In summary, therefore, $\mathrm{HaCaT}$ keratinocytes respond to UVB exposure by activating matriptase and generating ROS in a dose- and time-dependent manner. This suggests that the induction of matriptase zymogen activation by UVB may be linked with the generation of ROS since we have previously shown that $\mathrm{H}_{2} \mathrm{O}_{2}$, an agent commonly used to mimic endogenous ROS generation, can induce matriptase zymogen activation in $\mathrm{HaCaT}$ cells [23].

To further explore the relationship between ROS production and UVB-induced matriptase activation, we tested the ability of the ROS scavenger PBN (N-tert-Butyl- $\alpha$ phenylnitrone) (Figure 3A) and the reducing agent DTT (Figure 3B), to block UVB-induced matriptase zymogen activation. We found that UVB-induced matriptase zymogen activation was indeed suppressed by the presence of PBN and DTT in the culture medium in a dose-dependent 
manner (Figure 3). Taken together, these data suggest that ROS generation is likely involved in UVB-triggered matriptase zymogen activation in human keratinocytes.

The induction of ROS-mediated matriptase zymogen activation in $\mathrm{HaCaT}$ cells is not limited to the UVB portion of the spectrum. Exposure of the cells to UVA (Fig. 4A and B) and UVR (Fig. 4C and D) also induced matriptase zymogen activation and ROS generation. The effect of UV irradiation on matriptase appears to be primarily a post-translational event, involving matriptase zymogen activation followed by the rapid inhibition of the active matriptase by the formation of a complex with HAI-1. Neither the expression of matriptase of the inhibitor

HAI-1 appear to be significantly altered in acutely UV exposed cells. Collectively, the data from the cultured cell model indicates that UV irradiation, regardless of its wavelength, causes matriptase zymogen activation, and that this is likely mediated by ROS generation.

\section{Matriptase protects human keratinocytes from apoptotic cell death}

\section{caused by UV irradiation.}

We have recently shown that matriptase can protect cells from the toxicity associated with exposure to cadmium chloride, a transition metal that harms the cells through ROS generation and redox imbalance [20]. We, therefore, examined whether matriptase might be able to protect keratinocytes from the toxicity associated with UVB irradiation. To do this we used sub-lines of HaCaT cells in which matriptase levels had been suppressed by lentiviral delivery of a matriptase-targeting shRNA (MTP KD) or a non-targeting shRNA control (NT) [19]. Both HaCaT sublines were exposed to UVB irradiation (Figure 5), and as expected, an increase in matriptase zymogen activation was only observed in the non-target control cells and not in the 
matriptase knockdown HaCaT cells (Figure 5A, upper panel). The expression of HAI-1 (55-kDa), was not affected by matriptase knockdown (Figure 5A, middle panel) and the detection of the 120-kDa matriptase-HAI-1 complex indicates the level of matriptase zymogen activation (Figure $5 \mathrm{~A})$.

To examine the effect of matriptase knockdown on the toxicity caused by UVB, we assessed the UVB-induced apoptotic response of the cells by staining them with Hoechst dye and assessing the fraction of apoptotic cells by microscopy. UVB irradiation $\left(100 \mathrm{~mJ} / \mathrm{cm}^{2}\right)$ caused a significant increase in the number of apoptotic cells in both the NT and MTP KD cells when compared with their respective non-treatment controls. Interestingly, the percentage of apoptosis in the MTP KD cells (63.4\%) was, however, almost double that in the NT cells $(30.8 \%)$ (Figure 5B). This differential sensitivity to the induction of apoptosis was confirmed by a similar difference in the increased cleavage of PARP and in the activation of caspase 3 that was observed in the MTP KD keratinocytes versus the NT control cells (Figure 5C). These data suggest that matriptase expression and/or zymogen activation protect keratinocytes from apoptosis induced by acute UVB exposure.

Similar to data were obtained when the knockdown and control HaCaT sublines were exposed to UVA and UVR irradiation (Fig. 6). Enhanced induction of apoptosis was observed in UVA and UVR exposed matriptase knockdown cells compared to the NT control cells as assessed by the cleavage of PARP and caspase 3 (Fig. 6).

\section{Significant increase in matriptase zymogen activation in solar-}

\section{damaged human skin.}


To further investigate the activation of matriptase in the context of natural solar exposure, we examined the status of matriptase zymogen activation in human skin samples exhibiting the signs of chronic solar-damage. Six human skin specimens were excised from the perilesional solar-damaged skin of lesions removed from patients. These samples were frozen, sectioned, processed for histology and scored for matriptase staining as described in the methods section. The demographic details of the patients from which the skin was removed and the activated matriptase score for each specimen is shown in Table 1. Images of the histology and immunestaining from representative cases are shown in figure 7. The H\&E staining clearly shows elastin fiber changes characteristic for tissues exhibiting severe solar-damaged (Figure 7A and. The distribution and expression of total matriptase (data not shown) and HAI-1 (Figure 7 B, F) in these tissue was similar to that observed in normal skin samples in our previous studies [19,23], suggesting that there is no significant change in the expression of matriptase and its cognate inhibitor HAI-1 associated with chronic solar damage. In contrast, however, to the very low levels of activated matriptase that can be seen in about $40 \%$ of the samples of normal control skin examined [19], abundant staining for activated matriptase was observed in all six of the six solar-damaged skin cases examined (Figure $7 \mathrm{D}$ and H). In skin that also exhibited acanthosis (Figure $7 \mathrm{H}$ ), the activated matriptase detected was not limited to the basal layer but was also observed in the spinous layer, particularly at the cell-cell junctions (Figure $7 \mathrm{H}^{\prime}$ ) and was colocalized with HAI-1 (Figure 7 F'). Our data suggest that matriptase activity significantly increases in chronically solar damaged skin.

\section{Discussion}


Tightly controlled keratinocyte proliferation, apoptosis, and differentiation are required not only for epidermal homeostasis but also for epidermal tissue regeneration following insults to the skin. Although many molecular events involved in proliferation, apoptosis and differentiation are shared between epidermal tissue regeneration and epidermal homeostasis, the response to skin injury, by its nature, is rather different in that it requires that a rapid and robust molecular response be mounted immediately following the insult. The type 2 transmembrane serine protease matriptase is an activity that is involved in normal epidermal homeostasis. In normal resting skin, in spite of its abundant expression in the basal and spinous keratinocytes, matriptase is activated at very low levels. In contrast following UVB irradiation, high-level matriptase activation is rapidly induced in a manner that is characteristic of a response to tissue injury. It is interesting to note that in the context of chronic exposure to the insult, high levels of matriptase zymogen activation appear to be maintained. Thus, in addition to its role in normal keratinocyte proliferation and differentiation, matriptase activation follows the pattern of the rapid high-level response associated with the epidermal tissue regenerative response.

One of the important features of matriptase zymogen activation is that cleavage at the activation motif is not mediated by the action of another active protease, a common mechanism for zymogen activation of most serine proteases, and instead proceeds by an autoactivation mechanism. Autoactivation through the interaction of two matriptase zymogen molecules has been proposed to be the mechanism for the activational cleavage [27,28]. Matriptase autoactivation can be induced by several non-protease factors and can occur in a cell-type specific manner $[29,30]$. The chemical environment of cells, such as redox balance and acidity, also plays an important role of in the control of matriptase zymogen activation [20]. The kinetics of the induction of matriptase zymogen activation varies significantly among different activation 
inducers, ranging from minutes to hours. Other factors in the cellular chemical environment can also affect the onset of matriptase zymogen activation. For example, chloride can significantly attenuate matriptase zymogen activation induced by an acidic $\mathrm{pH}$ [20]. The dynamic features of the induction of matriptase zymogen activation has been also observed in our current study. In order to induce matriptase zymogen activation in human skin, a higher dose of UVB (300 $\left.\mathrm{mJ} / \mathrm{cm}^{2}\right)$ results in rapid matriptase activation kinetics $(10 \mathrm{~min})$. In contrast, at a lower dose (100 $\mathrm{mJ} / \mathrm{cm}^{2}$ ) it takes significantly longer ( 3 hours) for the induction of matriptase zymogen activation. This difference could be an effect of dose, or it may also be a function of the characteristics of the different experimental model used. The sensitivity of detection of the presence of activated matriptase in the different systems could also affect the time required for the appearance of activated matriptase. It could simply take much longer to accumulate sufficient activated matriptase to be detected by immunoblot analysis versus immunohistochemistry.

In addition to the interesting activation dynamics, the way that matriptase zymogen activation is induced is also important for its role in the epidermal response to UV irradiation. We have recently shown that matriptase zymogen activation can be rapidly induced by oxidative stress caused by the exposure of cells to the toxic metal cadmium chloride and to the oxidizer hydrogen peroxide $[20,23]$. The rapid generation and accumulation of cytosolic ROS in skin keratinocytes upon exposure to UVB irradiation may, therefore, represent the mechanism responsible for UVB-induced matriptase zymogen activation in the skin in our ex vivo sunburn model (Figure 1). Both UVA and UVR appear to also activate matriptase by a ROS-dependent manner and the cooperative oxidative stress from different spectrums of UV radiation may also 
contribute to the high levels of matriptase zymogen activation observed in skin showing signs of chronic solar damage (Figure 7). Acute UV irradiation has been known to induce leukocyte infiltration and so to cause inflammation [31], which results in the production of more ROS [32,33]. In addition, advanced glycation end products (AGEs) may provide an additional source of ROS to stimulate matriptase activation in the chronically solar damaged skins with actinic elastosis. AGE formation is a non-enzymatic process involving the modification of proteins by reducing sugars in an oxidative status. Previous studies show that the carboxymethyl lysine (CML), a major kind of AGE induced by solar irradiation, was highly expressed in the dermis of $\underline{\text { skin with actinic elastosis and the target proteins mainly exist in the extracellular matrix, such as }}$ elastin, collagen I and IV [34-36]. Under UVA exposure, these AGEs could cause the generation of large amounts of ROS [37]. Therefore, we suggested the sustained and increased accumulation of ROS from inflammation and/or AGEs provide a more oxidative environment to stimulate the robust activation of matriptase observed in chronically sun damaged skin.

Though increased matriptase zymogen activation may initially contribute to epidermal regeneration following UV irradiation, it is likely that adverse effects would result from chronically elevated matriptase zymogen activation in sun damaged skin. For example, increased matriptase activity may contribute to the abnormal degradation of elastin fibers mediated primarily by neutrophil-derived matrix metalloproteases (MMPs) and elastases in the dermis $[31,38,39]$. Persistent high-level matriptase zymogen activation would probably result in a proportion of the free active enzyme being shed from the keratinocytes and subsequently activating urokinase plasminogen activator (uPA), and thereby accelerating plasmin generation in the vicinity [40]. The increased plasmin generation can subsequently activate MMPs in the 
dermis [41]. Elevation of MMP activity has been observed to be induced in the skin by exposure to UV light and might contribute to the aberrant degradation of elastic fibers in the skins with actinic elastosis $[42,43]$. While enhanced proteolysis contributes to actinic elastosis, enhanced anti-proteolysis also plays a role in the complex process of photoaging. For example, elafin, a protease inhibitor, had been reported to be greatly accumulated in the UVA-exposed dermis. Elafin is crosslinked with elastin fibers to protect them from degradation by leukocyte elastase, resulting in the accumulation of these abnormal fibers in actinic elastosis [44]. It seems that UV irradiation causes multiple imbalances in the regulation of proteolysis in skin, some of which through increased proteolysis contribute to abnormal degradation of elastic fibers, whereas other, which increase protease inhibitors, contribute to the accumulation of abnormal elastic fibers. While the expression levels of matriptase and its cognate inhibitor HAI-1 may not be changed by chronic UV irradiation, the induction of matriptase activation is closely followed by shedding of active matriptase and HAI-1, some of which might find their way to the dermis and participate in the turnover of extracellular matrix proteins in the solar damaged skins.

Our data suggest that acute ultraviolet irradiation induces matriptase activation, which might protect keratinocytes from cell death. Heavy exposure to UV irradiation induces apoptosis of keratinocyte by direct DNA damage and/or ROS accumulation. Though this effect has been thought of as a positive selection to remove unhealthy and un-repaired cells to suppress tumorigenesis of the skin [45], excessive apoptotic cells could directly lead to impairment of the skin tissue to affect the quality of life [46]. Therefore, the protective effect of matriptase in UVexposed keratinocytes might play an important role to balance the side effect of heavy apoptosis in acute reaction phase. Chronically uncontrolled matriptase activity could also contribute to the 
development and progression of squamous cell carcinomas caused by UV irradiation.

Matriptase has been show to possess potent oncogenic activity when modestly overexpressed relative to its cognate inhibitor HAI-1in the skin of a transgenic mouse model [21]. Much of the matriptase oncogenic activity is thought to be attributed to its role on the activation of hepatocyte growth factor (HGF) [47] and subsequently the induction of c-Met signaling [48]. Thus, elevated matriptase zymogen activation in sun damaged skin could have a devastating effect.

Furthermore, the anti-apoptotic effect of matriptase demonstrated in this study could further enhance its role in the development of squamous cells carcinoma in sun damaged skin.

MiRNAs, small non-coding RNA, can bind to 3'-UTR regions of target mRNA resulting in the inhibition of protein production via RNA degradation or translational repression and have been linked to common chronic inflammatory skin disorders, such as psoriasis and atopic eczema [49]. Recently, this post-transcriptional regulation of miRNA had been shown to be involved in many cellular responses post UV exposure, such as apoptosis, inflammation, and carcinogenesis in skin [50]. Many miRNAs in skin might have been changed by UV irradiation and interestingly, UV of different wavelength ranges may cause different alterations in miRNA profile [51]. In addition, the changeable expression of some miRNA was time-dependent after UV response [52]. For example, miR-24, known to regulate cell cycle, was up-regulated 4 hours after UVB exposure and its levels remains up to 24 hours. Let-7a, a miRNA for cell proliferation, was also up-regulated 4 hours post UVB irradiation but its levels return to normal after 24 hours [52]. These lines of evidences seemingly indicate a vital role for miRNA in UVinduced skin responses. In human breast cancer cells, matriptase is a putative target gene of miR27b. The role of matriptase in cell migration and invasion can be regulated by miR-27b [53]. In 
human kerationcytes, miR-27b is up-regulated 4 hours after UVB irradiation [52]. The increased miR-27b might, however, not alter the levels of matriptase protein nor the zymogen activation.

In conclusion, our current study shows that matriptase could be a part of an early defense mechanism for dealing with the consequences of UV irradiation, which induces rapid matriptase zymogen activation through the generation and accumulation of ROS. Matriptase could protect the keratinocytes from apoptotic cell death and may contribute to epidermal regeneration after sun damage by virtue of its role in keratinocyte proliferation and early differentiation. While the pathophysiological role of the elevated matriptase zymogen activation in chronic sun damaged skin remains to be further investigated, uncontrolled matriptase activity could have adverse effects in the skin.

\section{Acknowledgement}

This study was supported by National Cancer Institute (NCI) Grants RO1 CA 123223 (to M. Johnson and C.-Y. Lin), Taiwan Department of Defense Grant 103-M017, MAB-104-28 (to C.-J. Chen), and 103-M018, MAB-104-26 (to J.-K. Wang), and Taipei Institute of Pathology Grant TIP10205 (to C.-H. Lai). 


\section{Reference List}

[1] Kulms D, Schwarz T. Independent contribution of three different pathways to ultraviolet-Binduced apoptosis. Biochem Pharmacol 2002;64:837-41.

[2] Jans J, Garinis GA, Schul W, van OA, Moorhouse M, Smid M, et al. Differential role of basal keratinocytes in UV-induced immunosuppression and skin cancer. Mol Cell Biol 2006 26: 8515-26.

[3] Bernerd F, Asselineau D. Successive alteration and recovery of epidermal differentiation and morphogenesis after specific UVB-damages in skin reconstructed in vitro. Dev Biol 1997;183:123-38.

[4] Eckert RL. Structure, function, and differentiation of the keratinocyte. Physiol Rev 1989; 69:1316-46.

[5] Elias PM. Skin barrier function. Curr Allergy Asthma Rep 2008;8:299-305.

[6] Hart TC, Hart PS, Bowden DW, Michalec MD, Callison SA, Walker SJ, et al. Mutations of the cathepsin C gene are responsible for Papillon-Lefevre syndrome. J Med Genet 1999;36:8817.

[7] Toomes C, James J, Wood AJ, Wu CL, McCormick D, Lench N, et al. Loss-of-function mutations in the cathepsin $\mathrm{C}$ gene result in periodontal disease and palmoplantar keratosis. Nat Genet 1999;23:421-4.

[8] Egberts F, Heinrich M, Jensen JM, Winoto-Morbach S, Pfeiffer S, Wickel M, et al. Cathepsin $\mathrm{D}$ is involved in the regulation of transglutaminase 1 and epidermal differentiation. J Cell Sci 2004;117:2295-307.

[9] Roth W, Deussing J, Botchkarev VA, Pauly-Evers M, Saftig P, Hafner A, et al. Cathepsin L deficiency as molecular defect of furless: hyperproliferation of keratinocytes and pertubation of hair follicle cycling. FASEB J 2000;14:2075-86.

[10] List K, Haudenschild CC, Szabo R, Chen W, Wahl SM, Swaim W, et al. Matriptase/MTSP1 is required for postnatal survival, epidermal barrier function, hair follicle development, and thymic homeostasis. Oncogene 2002;21:3765-79.

[11] List K, Szabo R, Wertz PW, Segre J, Haudenschild CC, Kim SY, et al. Loss of proteolytically processed filaggrin caused by epidermal deletion of Matriptase/MT-SP1. J Cell Biol 2003;163:901-10.

[12] Leyvraz C, Charles RP, Rubera I, Guitard M, Rotman S, Breiden B, et al. The epidermal barrier function is dependent on the serine protease CAP1/Prss8. J Cell Biol 2005;170:487-96.

[13] Demerjian M, Hachem JP, Tschachler E, Denecker G, Declercq W, Vandenabeele P, et al. Acute modulations in permeability barrier function regulate epidermal cornification: role of caspase-14 and the protease-activated receptor type 2. Am J Pathol 2008;172:86-97. 
[14] Denecker G, Hoste E, Gilbert B, Hochepied T, Ovaere P, Lippens S, et al. Caspase-14 protects against epidermal UVB photodamage and water loss. Nat Cell Biol 2007;9:666-74.

[15] Chavanas S, Bodemer C, Rochat A, Hamel-Teillac D, Ali M, Irvine AD, et al. Mutations in SPINK5, encoding a serine protease inhibitor, cause Netherton syndrome. Nat Genet 2000;25:141-2.

[16] Nagaike K, Kawaguchi M, Takeda N, Fukushima T, Sawaguch A i, Kohama K, et al. Defect of Hepatocyte Growth Factor Activator Inhibitor Type 1/Serine Protease Inhibitor, Kunitz Type 1 (Hai-1/Spint1) Leads to Ichthyosis-Like Condition and Abnormal Hair Development in Mice. Am J Pathol 2008;173:1464-75.

[17] Zeeuwen PL, van Vlijmen-Willems IM, Hendriks W, Merkx GF, Schalkwijk J. A null mutation in the cystatin $\mathrm{M} / \mathrm{E}$ gene of ichq mice causes juvenile lethality and defects in epidermal cornification. Hum Mol Genet 2002;11:2867-75.

[18] Zeeuwen PL, van Vlijmen-Willems IM, Olthuis D, Johansen HT, Hitomi K, HaraNishimura I, et al. Evidence that unrestricted legumain activity is involved in disturbed epidermal cornification in cystatin M/E deficient mice. Hum Mol Genet 2004;13:1069-79.

[19] Chen YW, Wang JK, Chou FP, Wu BY, Hsiao HC, Chiu H, et al. Matriptase regulates proliferation and early, but not terminal, differentiation of human keratinocytes. J Invest Dermatol 2013;134:405-14.

[20] Wang JK, Teng IJ, Lo TJ, Moore S, Yeo YH, Teng YC, et al. Matriptase autoactivation is tightly regulated by the cellular chemical environments. PLoS One 2014; 9:e93899.

[21] List K, Szabo R, Molinolo A, Sriuranpong V, Redeye V, Murdock T, et al. Deregulated matriptase causes ras-independent multistage carcinogenesis and promotes ras-mediated malignant transformation. Genes Dev 2005;19:1934-50.

[22] El-Abaseri TB, Putta S, Hansen LA. Ultraviolet irradiation induces keratinocyte proliferation and epidermal hyperplasia through the activation of the epidermal growth factor receptor. Carcinogenesis 2006;27:225-31.

[23] Chen CJ, Wu BY, Tsao PI, Chen CY, Wu MH, Chan YL, et al. Increased matriptase zymogen activation in inflammatory skin disorders. Am J Physiol Cell Physiol 2011;300:C40615.

[24] Kulms D, Zeise E, Poppelmann B, Schwarz T. DNA damage, death receptor activation and reactive oxygen species contribute to ultraviolet radiation-induced apoptosis in an essential and independent way. Oncogene 2002;21:5844-51.

[25] Kleszczynski K, Ernst IM, Wagner AE, Kruse N, Zillikens D, Rimbach G, et al. Sulforaphane and phenylethyl isothiocyanate protect human skin against UVR-induced oxidative stress and apoptosis: role of Nrf2-dependent gene expression and antioxidant enzymes.

Pharmacol Res 2013;78:28-40. 
[26] Oberst MD, Chen LY, Kiyomiya KI, Williams CA, Lee MS, Johnson MD, et al. Hepatocyte growth factor activator inhibitor 1 (HAI-1) regulates activation and expression of matriptase, a membrane-bound serine protease. Am J Physiol Cell Physiol 2005;289:C462-70.

[27] Oberst MD, Williams CA, Dickson RB, Johnson MD, Lin CY. The activation of matriptase requires its noncatalytic domains, serine protease domain, and its cognate inhibitor. J Biol Chem 2003;278:26773-9.

[28] Xu H, Xu Z, Tseng IC, Chou FP, Chen YW, Wang JK, et al. Mechanisms for the control of matriptase activity in the absence of sufficient HAI-1. Am J Physiol Cell Physiol 2012;302:C453-62.

[29] Benaud C, Oberst M, Hobson JP, Spiegel S, Dickson RB, Lin CY. Sphingosine 1phosphate, present in serum-derived lipoproteins, activates matriptase, J Biol Chem 2002;277:10539-46.

[30] Kiyomiya KI, Lee MS, Tseng IC, Zuo H, Barndt RJ, Johnson MD, et al. Matriptase activation and subsequent shedding with HAI-1 is induced by steroid sex hormones in human prostate cancer cells, but not in breast cancer cells. Am J Physiol Cell Physiol 2006; 291:C40-9.

[31] Rijken F, Bruijnzeel PL. The pathogenesis of photoaging: the role of neutrophils and neutrophil-derived enzymes. J Investig Dermatol Symp Proc 2009;14:67-72.

[32] Katiyar SK, Mukhtar H. Green tea polyphenol (-)-epigallocatechin-3-gallate treatment to mouse skin prevents UVB-induced infiltration of leukocytes, depletion of antigen-presenting cells, and oxidative stress. J Leukoc Biol 2001;69:719-26.

[33] Robinson JM. Reactive oxygen species in phagocytic leukocytes. Histochem Cell Biol 2008;130:281-97.

[34] Gkogkolou P, Bohm M. Advanced glycation end products: Key players in skin aging?. Dermatoendocrinol 2012;4:259-70.

[35] Yoshinaga E, Kawada A, Ono K, Fujimoto E, Wachi H, Harumiya S, et al. N(varepsilon)(carboxymethyl)lysine modification of elastin alters its biological properties: implications for the accumulation of abnormal elastic fibers in actinic elastosis. J Invest Dermatol 2012;132:315-23.

[36] Jeanmaire C, Danoux L, Pauly G. Glycation during human dermal intrinsic and actinic ageing: an in vivo and in vitro model study. Br J Dermatol 2001;145:10-8.

[37] Masaki H, Okano Y, Sakurai H. Generation of active oxygen species from advanced glycation end-products (AGEs) during ultraviolet light A (UVA) irradiation and a possible mechanism for cell damaging. Biochim Biophys Acta 1999;1428:45-56.

[38] Quan T, Qin Z, Xia W, Shao Y, Voorhees JJ, Fisher GJ. Matrix-degrading metalloproteinases in photoaging. J Investig Dermatol Symp Proc 2009;14:20-4. 
[39] Tsukahara K, Nakagawa H, Moriwaki S, Takema Y, Fujimura T, Imokawa G. Inhibition of ultraviolet-B-induced wrinkle formation by an elastase-inhibiting herbal extract: implication for the mechanism underlying elastase-associated wrinkles. Int J Dermatol 2006;45:460-8.

[40] Chen YW, Xu Z, Baksh AN, Wang JK, Chen CY, Swanson R, et al. Antithrombin Regulates Matriptase Activity Involved in Plasmin Generation, Syndecan Shedding, and HGF Activation in Keratinocytes. PLoS One 2013;8:e62826.

[41] Davis GE, Pintar Allen KA, Salazar R, Maxwell SA. Matrix metalloproteinase-1 and -9 activation by plasmin regulates a novel endothelial cell-mediated mechanism of collagen gel contraction and capillary tube regression in three-dimensional collagen matrices. J Cell Sci 2001;114:917-30.

[42] Fisher GJ, Wang ZQ, Datta SC, Varani J, Kang S, Voorhees JJ. Pathophysiology of premature skin aging induced by ultraviolet light. N Engl J Med 1997;337:1419-28.

[43] Ohnishi Y, Tajima S, Akiyama M, Ishibashi A, Kobayashi R, Horii I. Expression of elastinrelated proteins and matrix metalloproteinases in actinic elastosis of sun-damaged skin. Arch Dermatol Res 2000;292:27-31.

[44] Muto J, Kuroda K, Wachi H, Hirose S, Tajima S. Accumulation of elafin in actinic elastosis of sun-damaged skin: elafin binds to elastin and prevents elastolytic degradation. J Invest Dermatol 2007; 127:1358-66.

[45] Kulms D, Schwarz T. Molecular mechanisms of UV-induced apoptosis. Photodermatol Photoimmunol Photomed 2000;16:195-201.

[46] Van LA, Claerhout S, Garmyn M, Agostinis P. The sunburn cell: regulation of death and survival of the keratinocyte. Int J Biochem Cell Biol 2005;37:1547-53.

[47] Lee SL, Dickson RB, Lin CY. Activation of hepatocyte growth factor and urokinase/plasminogen activator by matriptase, an epithelial membrane serine protease. J Biol Chem 2000;275:36720-5.

[48] Szabo R, Rasmussen AL, Moyer AB, Kosa P, Schafer JM, Molinolo AA, et al. c-Metinduced epithelial carcinogenesis is initiated by the serine protease matriptase. Oncogene 2011;30:2003-16.

[49] Sonkoly E, Stahle M, Pivarcsi A. MicroRNAs: novel regulators in skin inflammation. Clin Exp Dermatol 2008;33:312-15.

[50] Syed DN, Khan MI, Shabbir M, Mukhtar H. MicroRNAs in skin response to UV radiation. Curr Drug Targets 2013;14:1128-34.

[51] Kraemer A, Chen IP, Henning S, Faust A, Volkmer B, Atkinson MJ, et al. UVA and UVB irradiation differentially regulate microRNA expression in human primary keratinocytes. PLoS One 2013;8:e83392. 
[52] Zhou BR, Xu Y, Permatasari F, Liu WL, Li W, Guo XF, Huang QH, et al. Characterization of the miRNA profile in UVB-irradiated normal human keratinocytes. Exp Dermatol 2012;21:317-9.

[53] Wang Y, Rathinam R, Walch A, Alahari SK. ST14 (suppression of tumorigenicity 14) gene is a target for miR-27b, and the inhibitory effect of ST14 on cell growth is independent of miR27b regulation. J Biol Chem 2009;284:23094-106. 


\section{Figure legends}

Figure 1. Increased activated matriptase in normal human skins after UVB irradiation. Normal human skin specimens were exposed to UVB $\left(300 \mathrm{~mJ} / \mathrm{cm}^{2}\right)$ irradiation - or sham exposed and were maintained at $37^{\circ} \mathrm{C}$ for 10 minutes, frozen in OTC and sections prepared. The serial sections were stained with hematoxylin and eosin (HE; A, E) or immunostained with $\operatorname{IgG}$ as a negative control (B, F), or with mAb M24 for total MTP (matriptase) (C, G), or mAb M69 for activated MTP (D, H) and high magnification images (D', H'). Nuclei were counterstained blue with hematoxylin. Scale bar $=100 \mu \mathrm{m}$. Representative images of the staining are presented.

\section{Figure 2. UVB causes matriptase activation and induces ROS accumulation in keratinocytes.}

HaCaT human keratinocytes were incubated for $6 \mathrm{hr}$ after the indicated dose of UVB irradiation, (left panels), or incubated for the indicated times post UVB irradiation $\left(100 \mathrm{~mJ} / \mathrm{cm}^{2}\right)($ right panels). The cells were analyzed by Western blotting (A and C) for activated matriptase (activated MTP) and total matriptase (total MTP) and by $\mathrm{H}_{2}$ DCF-DA detection for cytosolic ROS (B and D). Data shown are the mean \pm SD of eight and four independent experiments (B and $\mathrm{D}$, respectively). The significances are compared with each control $\left(0 \mathrm{hr}\right.$ or $\left.0 \mathrm{~mJ} / \mathrm{cm}^{2}\right)$. * $\mathrm{P}$ $\leqq 0.001$. ROS, reactive oxygen species.

\section{Figure 3. ROS is involved in UVB-induced matriptase activation.}

HaCaT human keratinocytes were exposed to UVB irradiation $\left(100 \mathrm{~mJ} / \mathrm{cm}^{2}\right)$ and incubated for 6 $\mathrm{hr}$ in the absence or the presence of various concentrations of PBN (A) or DTT (B) as indicated. 
The control and the treated cells were analyzed by Western blotting for activated matriptase (activated MTP) and total matriptase (total MTP).

\section{Figure 4. Both UVA and UVR cause matriptase zymogen activation via ROS generation} HaCaT cells were exposed to UVA or UVR irradiation at the indicated doses and then incubated in the absence or presence of DTT $(2 \mathrm{mM})$ for $6 \mathrm{hr}$. The levels of activated matriptase (activated MTP), total matriptase (total MTP), and HAI-1 were analyzed by Western blotting (A, C) and the levels of cytosolic ROS $4 \mathrm{hr}$ post irradiation was determined by the $\mathrm{H}_{2}$ DCF-DA method (B, D). Data shown in panel $(B, D)$ are the mean $\pm \mathrm{SD}$ of three independent experiments. The symbol * are compared with each negative control (UVA: $0 \mathrm{~J} / \mathrm{cm}^{2}$ and UVR: $0 \mathrm{~mJ} / \mathrm{cm}^{2}$, respectively) and the symbol ${ }^{\#}$ are compared with each condition of the highest dose treatment (UVA: $5 \mathrm{~J} / \mathrm{cm}^{2}$ and UVR: $80 \mathrm{~mJ} / \mathrm{cm}^{2}$ ) in the absence of DTT. * and ${ }^{\#} \mathrm{P} \leqq 0.001$. ROS, reactive oxygen species.

\section{Figure 5. Matriptase suppresses UVB-induced apoptosis in human karatinocytes.}

Both non-target control (NT) and matriptase knockdown (MTP KD) HaCaT keratinocytes were exposed to the indicated doses of UVB and were analyzed 6 hours after the exposure by Western blotting for activated matriptase (activated MTP), HAI-1, and actin (A). The ratio of apoptotic cells in both NT and MTP KD HaCaT cells $24 \mathrm{hr}$ after UVB or sham exposure was determined by counting cells stained with Hoechst 33258 (B). Lysates were also assayed for PARP, or caspase 3 cleavage by immunoblot analysis (C). Data shown in panel (B) are the mean \pm SD of five independent experiments. $* \mathrm{P} \leqq 0.001$. The arrows mark the cleaved form of indicated proteins. 
Figure 6. HaCaT cells with reduced matriptase expression are more susceptible to apoptosis induced by UVA or UVR.

Both non-target control (NT) and matriptase knockdown (KD) HaCaT keratinocytes were irradiated by sham control or UV irradiation (UVA: $7 \mathrm{~J} / \mathrm{cm}^{2}$ or UVR: $80 \mathrm{~mJ} / \mathrm{cm}^{2}$ ) and then incubated for $6 \mathrm{hr}$ at $37^{\circ} \mathrm{C}$ (A and B). Cell lysates were analyzed by Western blot for activated matriptase (activated MTP), PARP, cleaved-caspase 3, and actin by immunoblot analysis. The arrows mark the cleaved form of indicated proteins.

\section{Figure 7. The expression of activated matriptase in solar damaged skin.}

Frozen sections of six samples of solar damaged human skin were stained with hematoxylin and eosin (HE, A, E) or immunostained with IgG for negative control (C, G), mAb M19 for HAI-1 (B, F), and mAb M69 for activated matriptase (activated MTP) (D, H) in serial section. Staining of HAI-1 (B', F') and activated MTP (D', H') are also presented at high magnification. Nuclei were counterstained blue with hematoxylin. The skin tissue is composed of two primary layers, epidermis (Epi) and dermis (Der), respectively. The indicator for solar-damaged skin, elastin fiber degradation, is marked with arrows. Scale bar $=100 \mu \mathrm{m}$. 


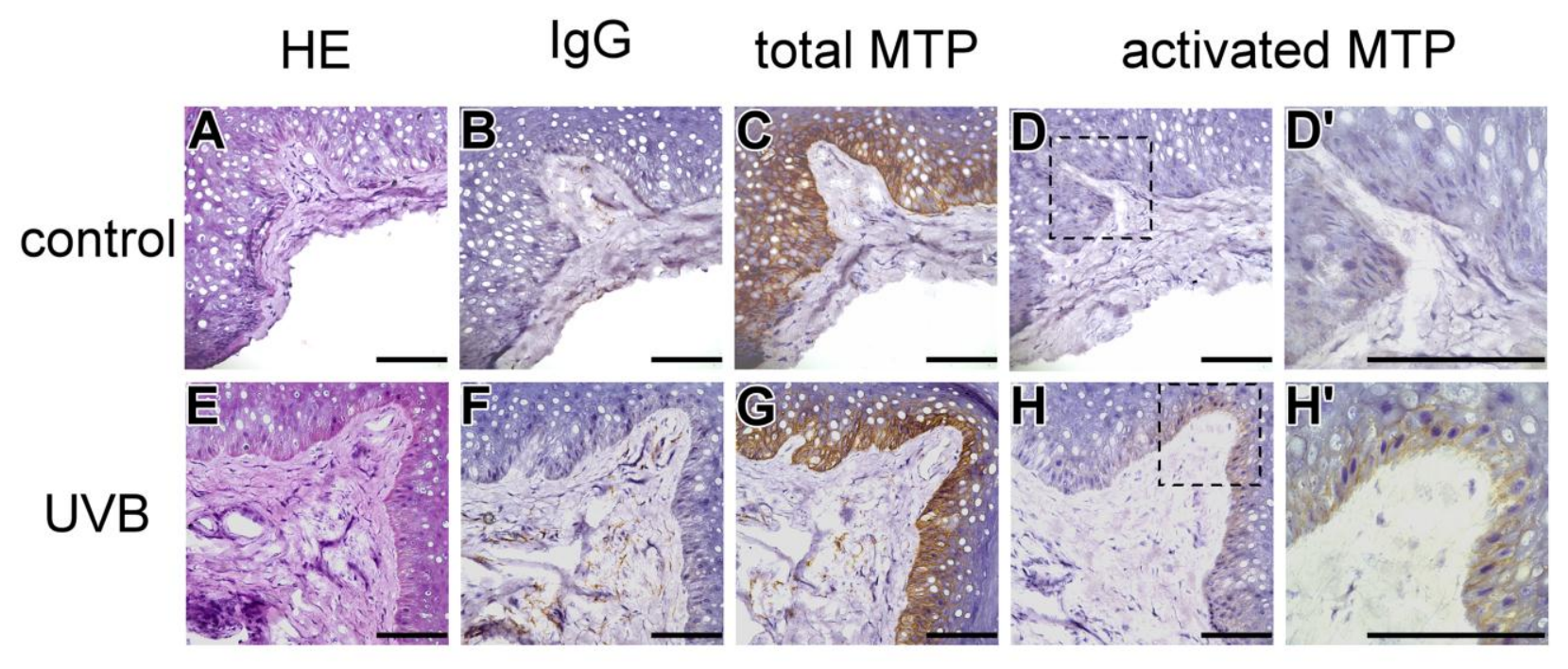



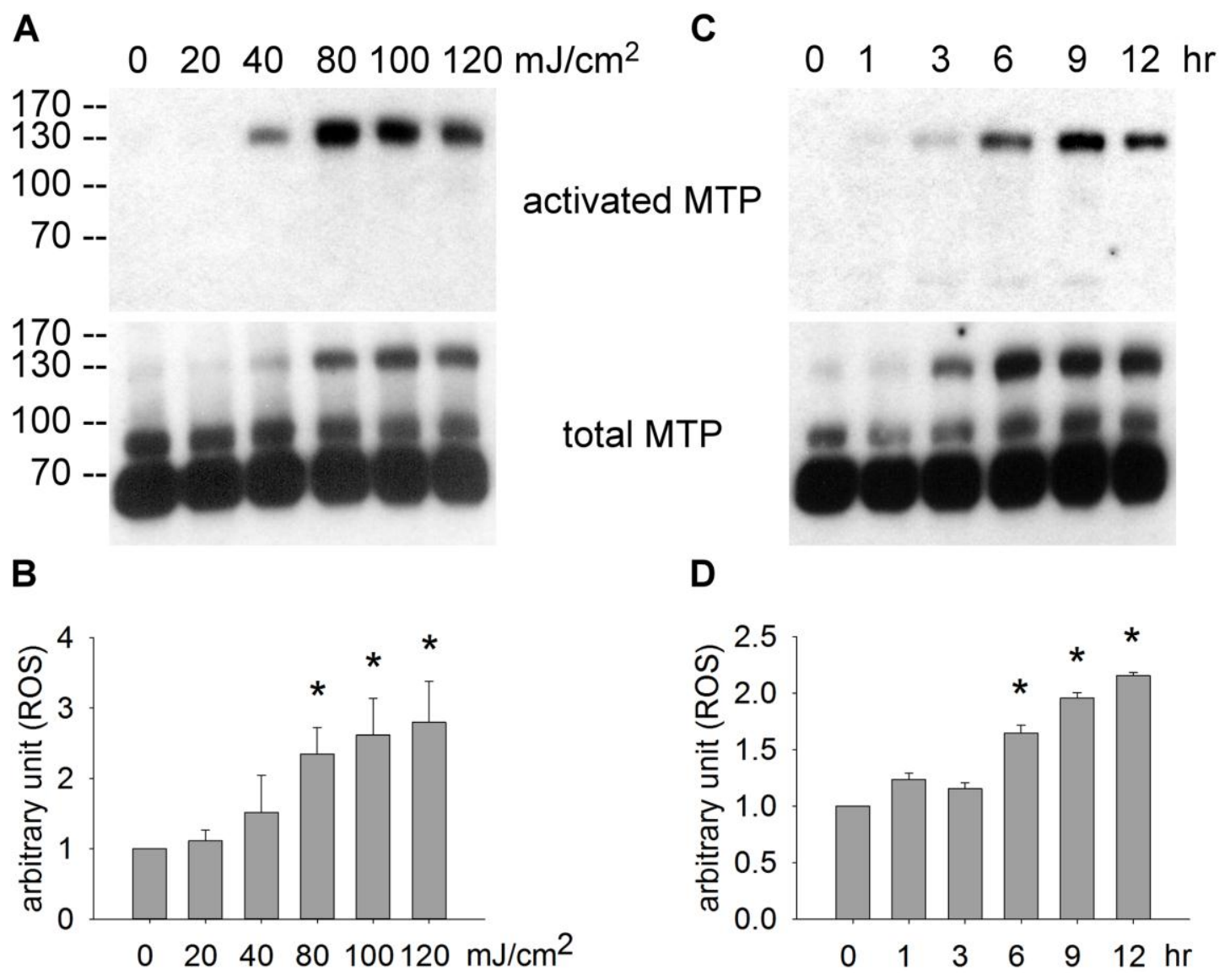


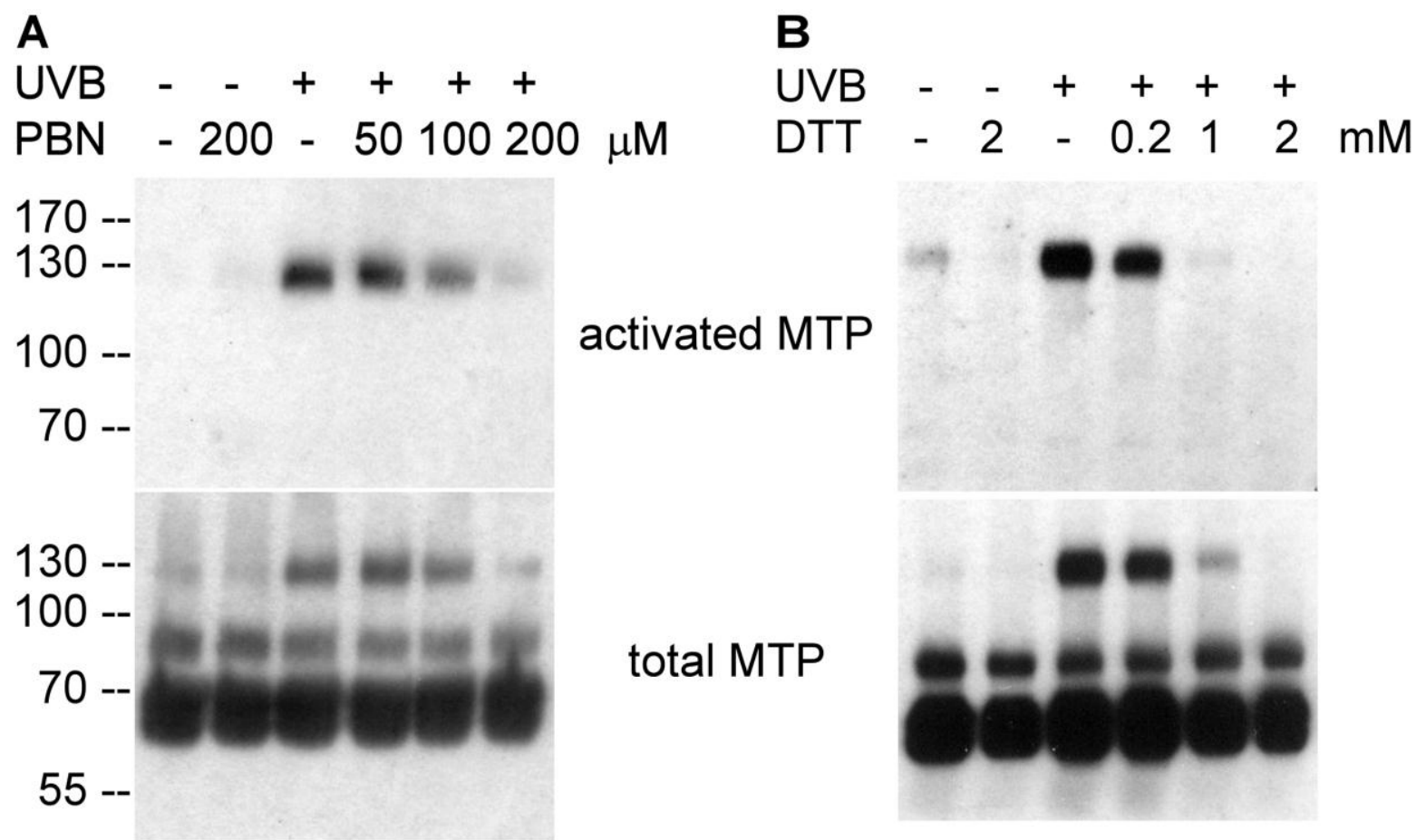


A C

$\begin{array}{llllllllllllll}\text { UVA } & 0 & 1 & 3 & 5 & 5 & \mathrm{~J} / \mathrm{cm}^{2} & \text { UVR } & 0 & 25 & 50 & 80 & 80 & \mathrm{~mJ} / \mathrm{cm}^{2}\end{array}$

DTT - - - + + DTT - - - $\quad+$

130-- $\approx=-\quad$ activated MTP

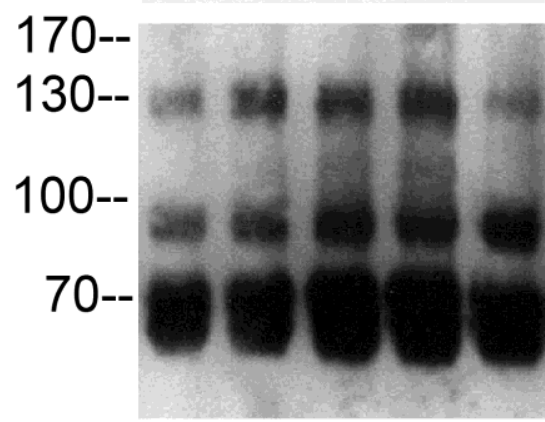

total MTP

wh ons

130--

100--

70--

HAI-1

55--

B

D
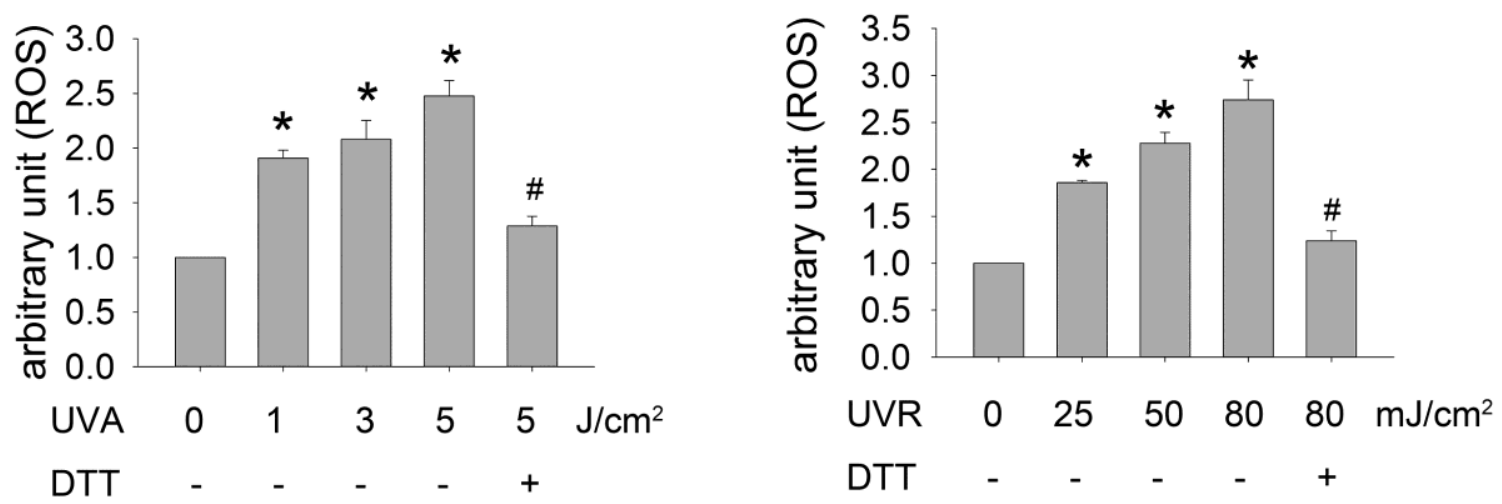


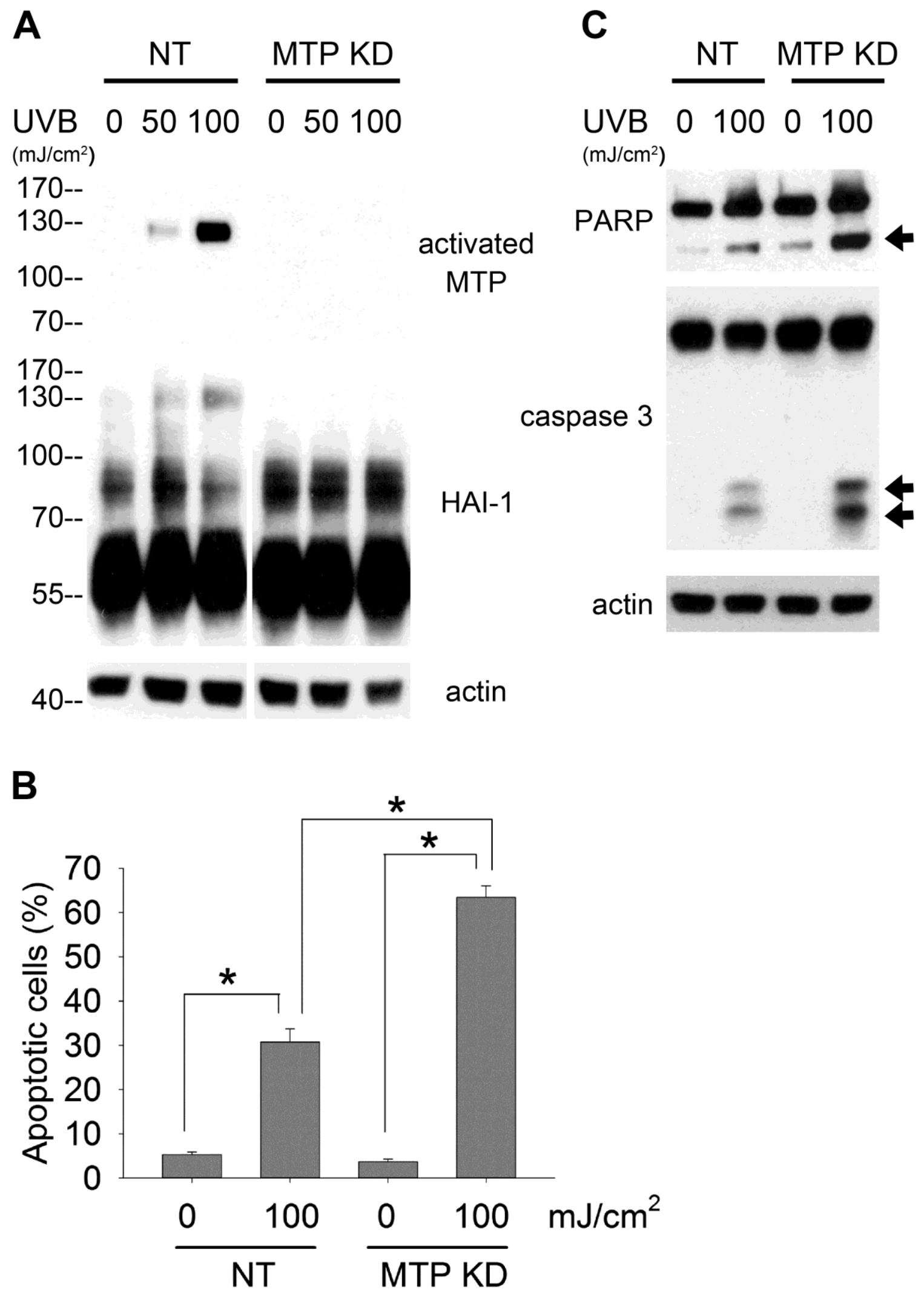


A

$$
\text { UVA } \frac{N T}{-+} \frac{K D}{-+}
$$

$$
\text { activated }
$$

MTP

PARP

cleaved-
casp 3

actin
B

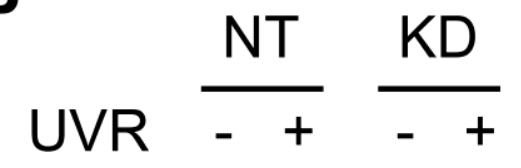

activated

MTP

PARP

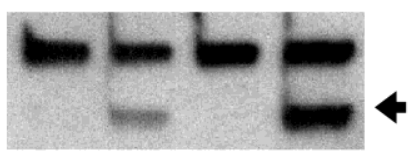

cleavedcasp 3

actin 

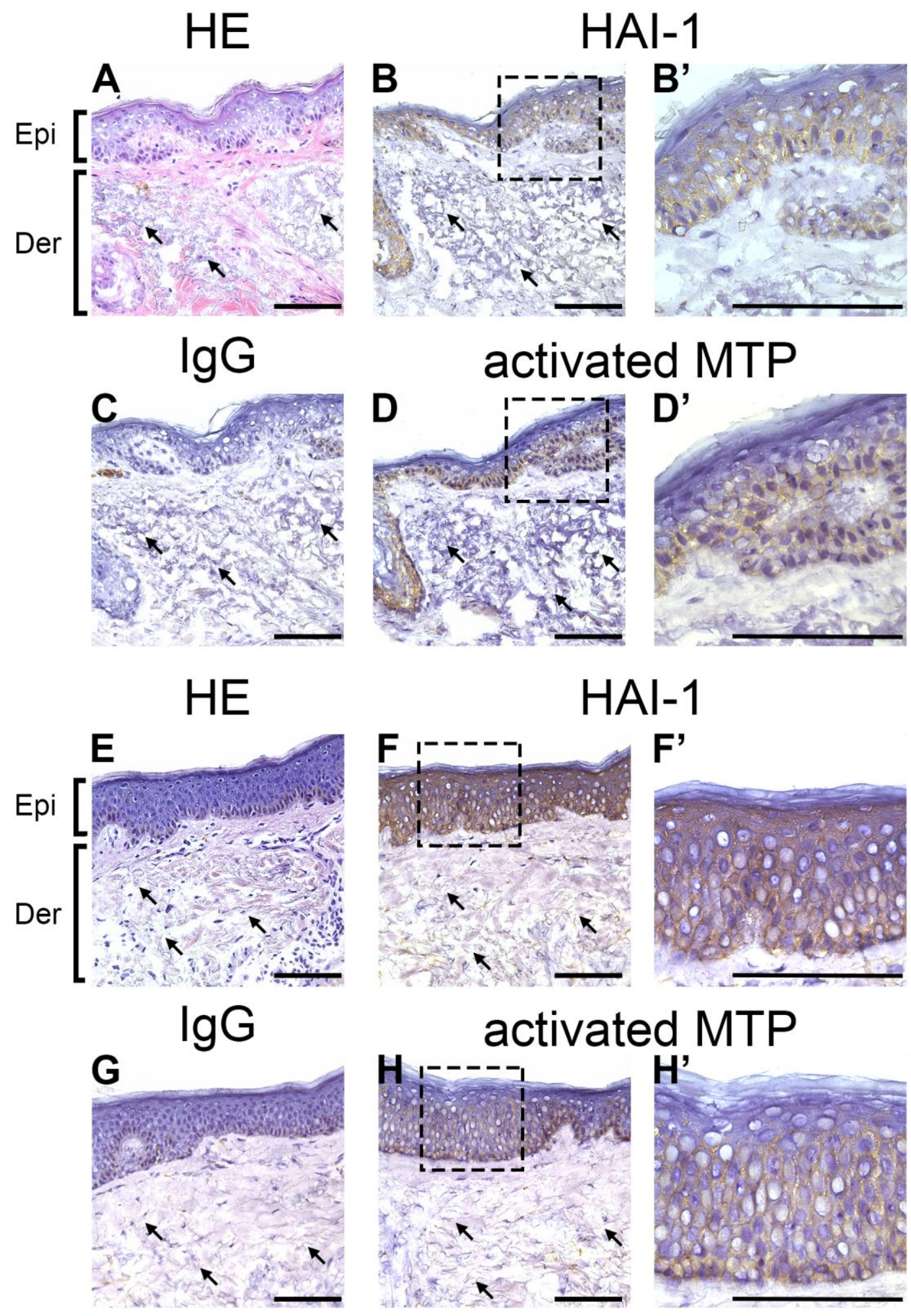


\begin{tabular}{lllc} 
Case no. & Age/sex & Site & $\begin{array}{c}\text { Reactivity with } \\
\text { M69 antibody }\end{array}$ \\
\hline 1 & $82 / M$ & Face & ++ \\
2 & $74 / F$ & Face & +++ \\
3 & $73 / F$ & Shin & + \\
4 & $57 / F$ & Face & + \\
5 & $48 / M$ & Nape & +++ \\
6 & $38 M$ & Face & ++ \\
\hline
\end{tabular}

Abbreviations: $F$, female; $M$, male.

${ }^{1}+++$, extremely positive; ++ , strongly positive; + , moderately positive.

Table 1. Immunoreactivity of actinic elastosis-expressed skins with monoclonal M69 antibody 